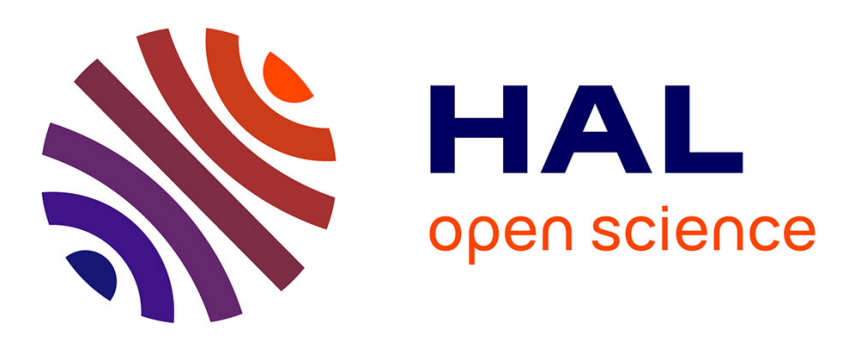

\title{
Stabilization of periodic discrete-time nonlinear systems
}

Mohamed Bensoubaya, Abdelhak Ferfera, Abderrahman Iggidr

\section{To cite this version:}

Mohamed Bensoubaya, Abdelhak Ferfera, Abderrahman Iggidr. Stabilization of periodic discretetime nonlinear systems. 36th IEEE Conference on Decision and Control, 1997, San Diego, California, United States. pp.3, 10.1109/cdc.1997.650760 . hal-01862875

\section{HAL Id: hal-01862875 \\ https://hal.inria.fr/hal-01862875}

Submitted on 29 Aug 2018

HAL is a multi-disciplinary open access archive for the deposit and dissemination of scientific research documents, whether they are published or not. The documents may come from teaching and research institutions in France or abroad, or from public or private research centers.
L'archive ouverte pluridisciplinaire HAL, est destinée au dépôt et à la diffusion de documents scientifiques de niveau recherche, publiés ou non, émanant des établissements d'enseignement et de recherche français ou étrangers, des laboratoires publics ou privés. 


\title{
Stabilization of Periodic Discrete-Time Nonlinear Systems
}

\author{
M. Bensoubaya \\ A. Ferfera \\ A. Iggidr \\ CONGE Project INRIA Lorraine \& University of Metz (URA-CNRS 399) \\ 57045 Metz cedex 01, France. e-mail:\{bensouba,ferfera,iggidr\}@loria.fr
}

\begin{abstract}
This paper provides sufficient conditions for stabilization of periodic discrete systems. These conditions are derived from a discrete version of a Theorem due to Krasovski for continuous-time systems. This tool allows to give a systematic design of time-varying stabilizing control for autonomous discrete systems that can not be stabilized by time-invariant feedback.
\end{abstract}

Keywords: Lyapunov function, stability, stabilization, time-varying feedback, periodic discrete systems.

\section{Introduction}

The aim of this article is to design stabilizer feedback for a class of periodic discrete-time nonlinear control systems. More precisely, we consider systems that can be written $x_{k+1}=F\left(x_{k}, u_{k}, k\right), F$ being time-periodic and the unforced system being Lyapunov stable. It is also assumed that there exists a positive definite periodic function $V(x, k)$ in such a way $V(F(x, 0, k), k+1)-V(x, k) \leq 0$. The paper is organized as follows. In Section 2, we give a discrete version of Krasovski theorem [6] and a sufficient condition under which the attractivity of the equilibrium point is guaranteed. In Section 3, this will be used to derive a stabilization result of periodic systems. As an application, we derive a sufficient condition for time-varying feedback stabilization for a class of autonomous systems that can not be stabilized by time-invariant feedback $[1,2,3,5]$. Due to the lack of space, the proofs are not reproduced here, but can be found in [4].

\section{Stability of periodic systems}

Let us consider a discrete nonlinear system $x_{k+1}=f\left(x_{k}, k\right), f(0, k)=0, x(k) \in \mathbb{R}^{n}, k \in \mathbb{N}$ We assume that $f$ is continuous and periodic with respect to $k$, i.e. there exists $T \in \mathbb{N}$ such that $f(x, k+T)=f(x, k), \forall(x, k) \in \mathbb{R}^{n} \times \mathbb{N}$. We shall denote by $x\left(k, x_{k_{0}}, k_{0}\right)$ the solution of (1) with initial state $x_{k_{0}}$ at initial time $k_{0}$. Let $a: \mathbb{R}^{+} \rightarrow \mathbb{R}^{+}$be a continuous function. We shall say that $a$ belongs to $\mathcal{K}$ if it is strictly increasing, vanishes at zero and $\lim _{r \rightarrow+\infty} a(r)=+\infty$.

Theorem 1 Suppose there exists a continuous function $V: \mathbb{R}^{n} \times \mathbb{N} \rightarrow \mathbb{R}$, periodic in $k$ with period $T \geq 2$ such that for some $a \in \mathcal{K}, \forall x \in \mathbb{R}^{n}, \forall k \in \mathbb{N}$ :

(1) $V(x, k) \geq a(\|x\|), V(0, k)=0$.
(2) $\Delta V(x, k)=V(f(x, k), k+1)-V(x, k) \leq 0$.

(3) The set $\left\{(x, k) \in \mathbb{R}^{n} \times \mathbb{N}: \Delta V(x, k)=0\right\}$ contains no complete solution of (1) except the trivial one.

Then the origin is globally uniformly asymptotically stable.

For the sequel, we define for all $0 \leq k \leq T$ a function $\hat{f}_{k}$ in the following way : $\hat{f}_{k}{ }^{k}(x)=x$ and by induction $\hat{f}_{k}^{p+1}(x)=f\left(\hat{f}_{k}^{p}(x), p\right)$ for all $p \geq k$. Actually $\hat{f}_{k}^{p}(x)$ is nothing but the value at time $p$ of the solution of (1) with initial state $x$ at initial time $k$. With this notation we can prove the following result which gives a sufficient condition to get the condition (3) of the above theorem.

Proposition 1 Suppose (1) and (2) hold. If the set $\left\{x \in \mathbb{R}^{n}: V\left({\hat{f_{k}}}^{p+1}(x), p+1\right)-V\left(\hat{f}_{k}^{p}(x), p\right)=0, p \geq k\right\}$ is reduced to the origin for all $k=0, \ldots, T$, then assumption (3) of Theorem (1) is satisfied and so the origin is globally uniformly asymptotically stable.

\section{Stabilization}

Consider a discrete-time nonlinear control system

$$
x_{k+1}=F\left(x_{k}, u_{k}, k\right), F(0,0, k)=0, k \in \mathbb{N}
$$

where $x_{k} \in \mathbb{R}^{n}$ is the state at time $k$ and $u \in \mathbb{R}^{m}$ is the control. $F: \mathbb{R}^{n} \times \mathbb{R}^{m} \times \mathbb{N} \rightarrow \mathbb{R}$ is assumed to be continuous in $(x, u)$ and periodic with respect to time $k$ with period $T \geq 2$. The problem addressed here is the following: does a periodic feedback $u(x, k), u(0, k)=0$, exists such that the origin is a globally asymptotically stable equilibrium point for the closed-loop system

$$
x_{k+1}=F\left(x_{k}, u\left(x_{k}, k\right), k\right)
$$

We will give a sufficient condition under which such a feedback exists. To do this we need the following notations. For a Lyapunov function $V(x, k)$ which is $C^{2}$ in $x$ and $T$-periodic in $k$ let $\tilde{V}: \mathbb{R}^{n} \times \mathbb{R}^{m} \times \mathbb{N} \rightarrow \mathbb{R}$ and $\varphi: \mathbb{R}^{n} \times \mathbb{R}^{m} \times \mathbb{R}^{m} \times \mathbb{N} \rightarrow \mathbb{R}$ be defined by

$$
\begin{aligned}
\tilde{V}(x, u, k) & =V(F(x, u, k), k+1) \\
\varphi(x, u, v, k) & =\int_{0}^{1}(1-t) v^{T} \frac{\partial^{2} \tilde{V}}{\partial u^{2}}(x, t u, k) v d t
\end{aligned}
$$

Set $F_{0}(x, k)=F(x, 0, k)$. For a fixed $\eta>0$, let $K_{1}(x, k)$ and $K_{2}(x, k)$ be any $T$-periodic and continuous real valued functions satisfying for all $(x, k) \in \mathbb{R}^{n} \times \mathbb{N}$, $K_{1}(x, k)+K_{2}(x, k) \neq 0$ and 


$$
\begin{aligned}
& K_{1}(x, k) \geq \sup _{\|u\| \leq \eta,\|v\|=1}|\varphi(x, u, v, k)| \\
& K_{2}(x, k) \geq\left\|\frac{\partial V}{\partial x}\left(F_{0}(x, k), k+1\right) \frac{\partial F}{\partial u}(x, 0, k)\right\|
\end{aligned}
$$

and set

$$
K(x, k)=\frac{\eta}{\eta K_{1}(x)+K_{2}(x)}
$$

One can notice that $K$ is $T$-periodic with respect to $k$. Now, we can state the main result of this section :

Theorem 2 Assume that $x_{k+1}=F_{0}\left(x_{k}, k\right)$ is stable and there exists a $C^{2}$ Lyapunov function $V(x, k)$ $T$-periodic in $k$ such that $V$ and $F_{0}$ satisfy the assumptions (1) and (2) of Theorem (1). If for all $k \in\{0, \ldots, T\}$ the set

$$
\begin{aligned}
& \left\{x \in \mathbb{R}^{n}: V\left(\hat{F}_{0 k}^{p+1}(x), p+1\right)=V\left(\hat{F}_{0 k}^{p}(x), p\right),\right. \\
& \left.\quad \frac{\partial V}{\partial x}\left(\hat{F}_{0 k}^{p+1}(x), p+1\right) \frac{\partial F}{\partial u}\left(\hat{F}_{0 k}^{p}(x), 0, p\right)=0, p \geq k\right\}
\end{aligned}
$$

is reduced to the origin then, for any positive constant $\eta$, system (2) is globally asymptotically stabilizable by means of the continuous feedback law

$$
u=-K(x, k)\left(\frac{\partial V}{\partial x}\left(F_{0}(x, k), k+1\right) \frac{\partial F}{\partial u}(x, 0, k)\right)^{T}
$$

which satisfies

$$
\left\{\begin{array}{l}
\|u(x, k)\| \leq \eta \quad \forall(x, k) \in \mathbb{R}^{n} \times \mathbb{N} \\
u(0, k)=0 \quad \forall k \in \mathbb{N}, \\
u(x, k+T)=u(x, k) \quad \forall(x, k) \in \mathbb{R}^{n} \times \mathbb{N}
\end{array}\right.
$$

Now, we apply the above theorem to the following nonlinear control system

$$
x_{k+1}=x_{k}+\Phi\left(x_{k}, u_{k}\right)
$$

where $x \in \mathbb{R}^{n}, u \in \mathbb{R}^{m}$ and $\Phi: \mathbb{R}^{n} \times \mathbb{R}^{m} \rightarrow \mathbb{R}^{n}$ is a $\mathcal{C}^{2}$ function satisfying $\Phi(x, 0)=0, \forall x \in \mathbb{R}^{n}$. It is known that if the map $\Phi$ fails to be locally onto then (8) cannot be stabilized by means of continuous state feedback $u_{k}=u\left(x_{k}\right)$. In what follows we give a sufficient condition for system (8) to be stabilizable by a time-varying feedback $u_{k}=u\left(x_{k}, k\right)$.

Theorem 3 Assume that for all $1 \leq i, j \leq m$

$$
\frac{\partial^{2} \Phi_{i}}{\partial u_{1} \partial u_{j}}(x, u)=0, \forall(x, u) \in \mathbb{R}^{n} \times \mathbb{R}^{m}
$$

and that there exist a $C^{2}$ Lyapunov function $V(x, k)$ and a continuous real valued function $\alpha(x, k)$ which are $T$-peridic with respect to time $k$ such that $V$ and the function $F_{0}$ defined by $F_{0}(x, k)=x+\alpha(x, k) g_{1}(x)$ satify the conditions of theorem 2. Then, for any positive constant $\eta$, system (8) is globally asymptotically stabilizable by means of the continuous feedback

$u(x, k)=\nu(x, k)+\tilde{u}(x, k)$

$\nu(x, k)=[\alpha(x, k), 0, \ldots, 0]^{T}$

$\tilde{u}(x, k)=-K(x, k)\left[\frac{\partial V}{\partial x}\left(F_{0}(x, k), k+1\right) \frac{\partial \Phi}{\partial u}(x, \nu(x, k))\right]^{T}$

where $K(x, k)$ is got from (3)-(7) with

$$
\tilde{V}(x, u, k)=V(x+\Phi(x, \nu(x, k)+u), k+1)
$$

Since the conditions and the design of the control laws in theorem 3 make use of the functions $V$ and $\alpha$, it is natural to look for particular systems of the form (8) for which $V$ and $\alpha$ can be explicited. Setting

$$
g(x)=\left(g_{1}(x), \ldots, g_{m}(x)\right)=\frac{\partial \Phi}{\partial u}(x, 0)
$$

it turns out, as in continuous-time (see [7]), that if

$$
g_{1}=\frac{\partial \Phi}{\partial u_{1}}(x, 0)=\frac{\partial}{\partial x_{1}}
$$

it is actually possible to give an explicit design of $V$ and $\alpha$, and by the way of the control laws.

Hereafter, for all $x \in \mathbb{R}^{n}$, set $\bar{x}=\left(x_{2}, \ldots, x_{n}\right)^{T}$ and for a fixed integer $T \geq 2$ let

$$
\begin{aligned}
V(x, k) & =\frac{1}{2}\left(\left[x_{1}+h(\bar{x}, k)\right]^{2}+\|\bar{x}\|^{2}\right) \\
\alpha(x, k) & =-\frac{1}{2}\left[x_{1}-h(\bar{x}, k)\right]-h(\bar{x}, k+1)
\end{aligned}
$$

where $h$ is a $\mathcal{C}^{2}$ function satisfying $h(\bar{x}, k+T)=h(\bar{x}, k)$, $\forall(\bar{x}, k) \in \mathbb{R}^{n-1} \times \mathbb{N}$ and $h(0, k)=0, \forall k \in \mathbb{N}$. A possible choice for $h$ is $h(\bar{x}, k)=\psi(\bar{x}) H(k)$ where $\psi$ is a definite function with respect to $\bar{x}$, and $H(k)$ is a time periodic function with period $T$. So $V$ and $\alpha$ are $T$-periodic with respect to time $k$, and $V$ vanishes if and only if $x=0$. With these notations we have:

Proposition 2 Assume that (9) and (11) hold. If there exists a function $h$ as specified above such that for all $\bar{x} \in \mathbb{R}^{n-1}$,

$\operatorname{rank}\left\{\tilde{g}_{i}(h(\bar{x}, k), \bar{x}), 2 \leq i \leq m, 0 \leq k \leq T-1\right\}=n-1$ where $\tilde{g}_{i}(x)=\left(0, g_{i}^{2}(x), \ldots, g_{i}^{n}(x)\right)$, then, for any positive constant $\eta$, system (8) is globally asymptotically stabilizable by means of the feedback (10).

\section{References}

[1] R.W. Brockett. Asymptotic stability and feedback stabilization, in Differential Geometric Control Theory, Birkhauser, Boston, 1983, p. 181-191.

[2] M. Bensoubaya, A. Ferfera and A. Iggidr. Stabilization of discrete-time nonlinear systems. Comtes rendus de l'Academie des Sciences, Paris, Tome 321, Série I, No. 3, Août 1995, p. 371-374.

[3] M. Bensoubaya, A. Ferfera and A. Iggidr. Timevarying stabilizing feedback for a class of nonlinear discrete-time control systems. 34th $C D C$ (1995), p. 512-514.

[4] M. Bensoubaya, A. Ferfera and A. Iggidr. Stabilization of periodic discrete-time nonlinear systems. INRIA report, 1997.

[5] W. Lin and C.I. Byrnes Design of discrete-time nonlinear control systems via smooth feedback. IEEE Trans. Autom. Control, 39, No 11, November 1994, p. 2340-2346. 
[6] N. N. Krasovski. Problems of the theory of stability of motion. Stanford Univ. Press, Stanford, California 1963 ; translation of the Russian edition, Moskow, 1959.

[7] J.B. Pomet. Explicit design of time-varying stabilizing control laws for a class of controllable systems without drift Systems $\&$ Control Letters, (18), (1992), p. $147-158$. 